\title{
Man With Flu-like Symptoms
}

\author{
Sandarsh Kancherla, MD, Ankitkumar Patel, MD, MPH
}

\section{Case Presentation}

A 69 year-old male presents to the Emergency Department with complaints of malaise, myalgias, rhinorrhea, increased congestion, and occasional fevers for one week. The symptoms have gradually worsened over the past week, and Tylenol has minimally alleviated his symptoms. He denies exacerbating factors. He denies any trauma, shortness of breath, chest pain, or recent weight change. He has no sick contacts or recent travel history.

Past medical history is significant for coronary artery disease status post myocardial infarction and coronary artery bypass graft in 2000, hypertension, hyperlipidemia, and atrial fibrillation. Medications on admission include amiodarone, aspirin, atorvastatin, docusate, losartan, metoprolol succinate (XL), and warfarin. He does not smoke, drink alcohol, or use illicit drugs.

On admission, temperature was 99.7, blood pressure was 118/72, heart rate was 74 , respiratory rate was 18 , and oxygen saturation was $96 \%$ on room air. Physical examination was significant for dry mucosa and mild proximal muscle weakness. Initial labs on admission revealed an elevated creatinine of 1.9; CBC, UA, and LFTs were within normal limits. The patient was admitted with the diagnosis of upper respiratory tract infection with dehydration and started on intravenous fluids and supportive therapy for flu-like symptoms.

Approximately 3 days after admission, his rhinnorhea and congestion had resolved, but he complained of worsening myalgias. Physical exam at that time revealed worsening tenderness to palpation over his shoulder muscles and proximal lower extremities, as well as 4/5 muscle strength in his quadriceps and deltoids. He had difficulty standing from a seated position. Range of motion of all extremities was intact. At that time, it was revealed that his atorvastatin dose had been increased from $40 \mathrm{mg}$ to $80 \mathrm{mg}$ approximately 6 months ago as an outpatient. The CPK level drawn shortly thereafter was ${ }^{5,317}$, supporting a diagnosis of statin-induced myositis, which is a form of drug-induced myopathy. Upon discontinuing his atorvastatin, his symptoms improved, and CPK trended down until it was within normal limits.

\section{Discussion}

Drug-induced myopathy is among the most common causes of muscle disease. Symptoms range from mild myalgias with or without mild weakness, to chronic myopathy characterized by severe weakness, to massive rhabdomyolysis with acute renal failure. ${ }^{1}$

Drug-induced myopathy results from direct myotoxicity from lipid-lowering drugs and other agents such as alcohol, cocaine, glucocorticoids, antimalarials, and colchicine. ${ }^{2}$ Statins are considered effective and generally safe, but experience in clinical practice suggests that muscle side effects are relatively common; some of the side effects are severe enough to warrant discontinuation of statin therapy.

The mechanism by which statins cause muscle toxicity is not well understood. They inhibit the conversion of HMG-CoA to mevalonic acid, which is an important early step in cholesterol synthesis. Statins can also decrease the synthesis of coenzyme Q10 (CoQ10), which plays an important role in muscle cell energy production. 3 This pathway is thought to contribute tostatin-induced muscle injury.

The onset of muscle symptoms is usually within weeks to months after the initiation of statin therapy. Typically, the presentation of statin-induced myopathy is very non-specific. It has a very insidious onset and includes progressive weakness, generalized pain, and fatigue. Myalgias and weakness generally resolve, and serum creatinine kinase levels return to normal within days to weeks upon discontinuation of statin therapy.

The muscle symptoms in patients taking statin therapy can range from mild symptoms to severe symptoms. The incidence of mild myalgias, defined as muscle ache or weakness without increases in CPK levels, in patients taking statins ranges from 2 to 11 percent. ${ }^{4-5}$ Symptoms generally resolve within 2 to 3 weeks after stopping a statin. Myositis, defined as a serum CPK elevation more than 10 times normal in association with muscle symptoms, occurs in less than $0.5 \%$ of patients. ${ }^{4-5}$ Rhabdomyolysis resulting from severe muscle destruction is rarely seen and occurs in less than $0.1 \%$ of patients. $^{6}$ Rhabdomyolysis has primarily been seen when a statin is given concurrently with cyclosporine or gemfibrozil.?

The risk of muscle injury due to statins appears to vary among the different drug formulations. The risk of myopathy is lowest with pravastatin (less than $0.1 \%$ ) and fluvastatin. ${ }^{8}$ These two medications are also less likely to cause drug interactions since they are not extensively metabolized by CYP3A4. Patients with acute or chronic renal failure, obstructive liver disease, and hypothyroidism have increased susceptibility to statinassociated myopathy.

The risk of myopathy is much greater in patients who are also taking drugs that inhibit CYP3A4, must notably cyclosporine, gemfibrozil, macrolideantibiotics, itraconazole, and HIV protease inhibitors. These drugs increase the serum concentration of the statins. Initial studies of lovastatin and simvastatin showed clinically significant myopathy in 13 to $30 \%$ of patients taking cyclosporine. ${ }^{4}$ Approximately $5 \%$ of patients taking atorvastatin or lovastatin in addition to gemfibrozil experience significant myopathy. ${ }^{4,9}$ In contrast, pravastatin and fluvastatin, which are not metabolized by CYP3A4, do not appear to increase the risk 
of myopathy when given with cyclosporine. ${ }^{10,11}$ This is a very important consideration when treating transplant patients.

Routine monitoring of CPK levels is not recommended, but it is useful to obtain a baseline serum CPK before initiation of statin therapy for reference. Patients should be alerted to the potential side effects of statins and report new onset of myalgias or weakness to their physician. CPK levels may be abnormal in conditions such as hypothyroidism, trauma, and high impact sports. In general, a CPK level more than 10 times the upper limit of normal, in the absence of other clinical risk factors, is generally felt to be due to a statin. It is an indication to discontinue the medication. If a patient experiences muscle myopathy on a statin, with the exception of rhabdomyolysis, the statin should be discontinued until symptoms resolve. It is reasonable to consider restarting pravastatin or fluvastatin with careful monitoring. In the case of rhabdomyolysis, the statin should be discontinued indefinitely. If muscle toxicity occurs while on pravastatin or fluvastatin, a trial of CoQ10 supplementation may be initiated, although there is only limited evidence for its benefit. ${ }^{12}$

\section{References}

1. Zuckner,J. Drug-related myopathies. Theum Dis Clin North Am 1994; 20:1017

2. Lane, RJM, Mastaglia, FL. Drug-induced myopathies in man. Lancet 1978; 2:562

3. Ghirlanda, G, Oradei, A, Manto, A, et al. Evidence of plasma CoQ10-lowering effect by HMG-CoA reductase inhibitors: a double-blind, placebo-controlled study. J Clin Pharmacol 1993;33: 226.

4. Tobert, JA. Efficacy and long-term adverse effect pattern of lovastatin. Am J Cardiol 1988;62: 28J

5. Boccuzzi, SJ, Bocanegra, TS, Walker, JF, et al. Long-term safety and efficacy profile of simvastatin. Am J Cardiol 1991; 68:1127

6. Antons, KA, Williams, CD, Baker, SK, Phillips, PS. Clinical perspectives of statininduced rhabdomyolysis. Am J Med 2006; 119:400

7. Pierce, LR, Wysowski, DK, Gross, TP. Myopathy and rhabdomyolysis with lovastatin-gemfibrozil comgination therapy. JAMA 1990; 264:71

8. Graham, DJ, Staffa, JA, Shatin, D, et al. Incidence of hospitalized rhabdomyolysis in patients treated with lipid-lowering drugs. JAMA 2004; 292:2585

9. Duell, PB, Connor, WE, Illingworth, DR. Rhabdomyolysis after taking atorvastatin with gemfibrozil. Am F Cardiol 1998; 81:368

10. Kobashigawa, JA, Katznelson, S, Laks, H, et al. Effect of pravastatin on outcomes after cardiac transplantation. N Engl J Med 1995; 333:621

11. Schrama, YC, Hene, RJ, de Jonge, N, et al. Efficacy and muscle safety of fluvastatin in cyclosporine-treated cardiac and renal transplant recipients. Transplantation 1998; 66:1175

12. Koumis, T, Nathan, JP, Rosenberg, JM, Cicero, LA. Strategies for the prevention and treatment of statin-induced myopathy: is there a role for ubiquinone supplementation? Am J Health Syst Pharm 2004; 61:515

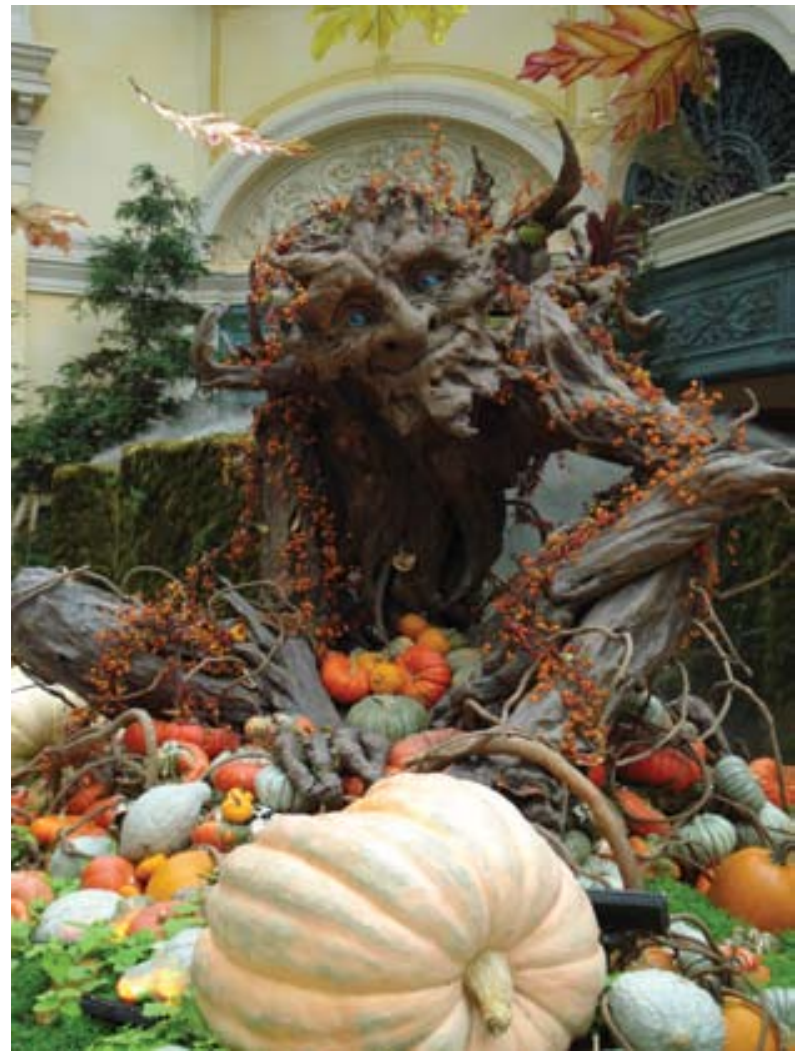

Photo courtesy of

Vaibhav Mehendiratta, MD 